\title{
Grassmann Tensor Renormalization Group Study of Lattice QED with Theta Term in Two Dimensions
}

\author{
Yuya Shimizu ${ }^{* a}$ and Yoshinobu Kuramashi ${ }^{b, c, a}$, \\ ${ }^{a}$ RIKEN Advanced Institute for Computational Science, Kobe, Hyogo 650-0047, Japan \\ ${ }^{b}$ Graduate School of Pure and Applied Sciences, University of Tsukuba, Tsukuba, Ibaraki \\ 305-8571, Japan \\ ${ }^{c}$ Center for Computational Sciences, University of Tsukuba, Tsukuba, Ibaraki 305-8577, Japan
}

\begin{abstract}
We apply the Grassmann tensor renormalization group to two-dimensional lattice QED. The phase structure with one-flavor of the Wilson fermion, especially including a case with the $\theta$ term at $\theta=\pi$ is studied. We present numerical results of finite size scaling analyses.
\end{abstract}

\section{References}

[1] Y. Shimizu and Y. Kuramashi, Phys. Rev. D 90 (2014) 014508.

[2] Y. Shimizu and Y. Kuramashi, Phys. Rev. D 90 (2014) 074503.

The 32nd International Symposium on Lattice Field Theory,

23-28 June, 2014

Columbia University New York, NY

\footnotetext{
*Speaker.

${ }^{\dagger}$ E-mail: yuya.shimizu@ riken.jp
} 